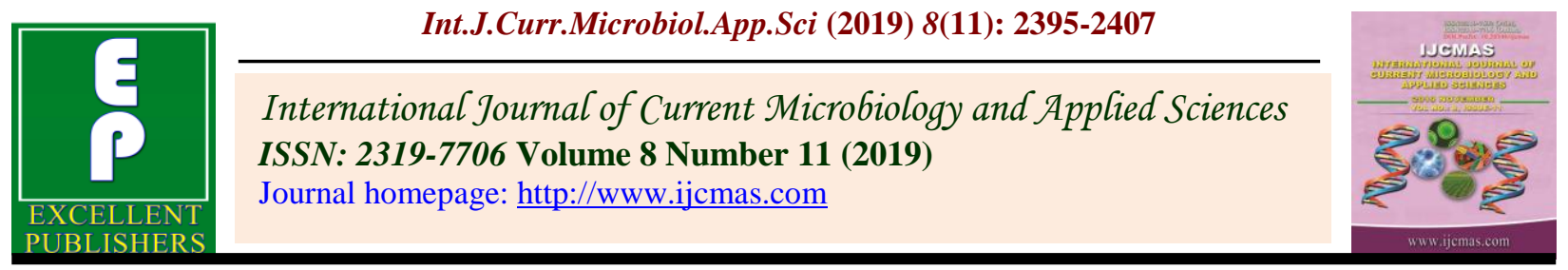

Review Article

https://doi.org/10.20546/ijcmas.2019.811.278

\title{
Screening of wheat (Triticum aestivum L.) genotypes for field performance and seed quality parameters
}

\author{
Vidyashree S. Naduvinamani*, S.B. Patil, Ashok S. Sajjan and M.D. Patil \\ Department of Seed Science and Technology, College of Agriculture, Vijayapura, \\ University of Agricultural Sciences, Dharwad-580005, Karnataka, India \\ *Corresponding author
}

\section{A B S T R A C T}

\begin{tabular}{|l|}
\hline K e y w o r d s \\
Wheat, \\
Morphological \\
traits, Physiological \\
traits, Genotypes
\end{tabular}

Keywords

Wheat,

Morphological traits, Physiological traits, Genotypes

Article Info

26 October 2019

10 November 2019
Wheat is an important cereal crop of world including India. The major problems of drought condition in India therefore wheat have a low productivity, so we would like to develop drought tolerant crop varieties through help of various verities. In which present study we have used twenty six wheat genotypes of diverse genetic background with popular cultivar of India. All the genotypes had screened accordingly morphological and physiological characterizations for drought condition. On the basis of morphological and physiological traits it was observed that genotypes C-306, BRW-3806, DBW-110, DBW-116, GW-477, HI1620, MACS-6695, MP-1331, NI-5439, NIAW-3212 and RW-5 performed better for drought induced conditions.

\section{Introduction}

Wheat (Triticum aestivum L.) is an annual, self-pollinated crop and belonging to Poaceae family (grasses). All over India, wheat crop is cultivated and considered as an essential staple food grain. It belongs to the sub-tribe Triticinae, which is recent origin of tribe Triticeae. This tribe contains about 35 genera including Thinopyrum, Triticum, Dasypyrum, Aegilops, Secale and Lophopyrum.

Area, productivity and production of wheat in Karnataka is 0.17 million hectare $(0.57 \%)$, $1,012 \mathrm{~kg}$ per ha and 0.17 million tonnes $(0.19$
$\%)$ respectively, during 2017-18 and wheat is being cultivated widely in Dharwad, Belgaum, Bijapur, Gulbarga and Bidar districts. In India, second major cereal crop is wheat after rice. The enhancement of productivity is in greater demand in building the country self adequate in food production of food. Under timely sown irrigated condition, semi-dwarf type genotypes are cultivated in a major part of the area (Joshi et al., 2007).

Hexaploid species of bread wheat is extensively cultivated throughout the world and followed by durum wheat of tetraploid species, which is the second most cultivated. 
In India mainly three kinds of wheat are grown viz., (i) Triticum aestivum (Common wheat) (ii) Triticum durum (durum wheat) and (iii) Triticum dicoccum (dicoccum wheat).

In general, $T$. aestivum and $T$. durum cultivars of wheat are more sensitive to high temperatures during crop growth and development. As compared to other wheat species, dicoccum has the capacity to withstand at higher temperature stress. The capability of dicoccum wheat for may be attributed to internal genetic makeup and morpho-physiological mechanisms.

Water is the essential input on which yields of field crops depends oftenly. Presently water provided for crop production is leap towards decline by future due to increasing demand of water for industrial and drinking purposes. Consequently, wheat is an essential food crop which is affected by water stress due to high requirement of water it will suffer the most if drought provides during crops period. This would cause a set back to the sustainability of wheat productivity. Additionally, insufficient accessibility of water and its distribution would always remain a limiting factor to attain expected yield in wheat. If the crop is exposed to water stress especially during crown root initiation stage at high temperature maximum impact on field is observed. Therefore, the heat stress and water are most important components that affect the productivity of wheat.

\section{Materials and Methods}

An experiment was conducted in rainout shelter, College of Agiculture, Vijayapura, Karnataka. The field experiment was laid out in randomized complete block design (RCBD) with two moisture regimes with three replications. After sowing, well water was provided both the treatments. At critical stages, no irrigation was applied for one set (low moisture stress) and crop was protected from rainfall.

\section{Morphological evaluation of wheat genotypes}

Observation of Morphological and physiological traits were recorded on randomly selected five plants from each tagged plants in each replication at deferent growth stage. The data was recorded for preharvest characters like plant height $(\mathrm{cm})$ from bottom of the plant from soil level to the base of the spike, productive tiller, days to 50 per cent flowering, days of maturity, length of spikelet $(\mathrm{cm})$ from tips of apical spikelet (excluding awns) to the bases or collar of ear, number of grains per spike (g), number of grains per spike, 1000 grain weight and yield per plant were recorded.

\section{Physiological evaluation of wheat genotypes}

The SPAD chlorophyll reading was taken at 30 and 60 DAS on which leaf using the SPAD meter on the green leaves in randomly selected five plants and the average relative chlorophyll content was worked out and expressed in number.

\section{Results and Discussion}

\section{Yield parameters}

The combined effect of two moisture regimes and wheat genotypes on grain yield was found significant. Under well water condition, the maximum grain yield was recorded in C-306 $(4.25 \mathrm{~g})$ followed by HI-1620 (1.76 g) whereas minimum yield for genotype DBW14 (3.44 g). Under water stress condition more reduction in grain yield was observed in HD2733 (1.39 g) genotype compared to other genotypes. The increased in grain yield per plant may be attributed higher plant height (Table 1 and Fig. 1), number of tillers per 
plant (Table 1 and Fig. 2), spike length (Table 1 and Fig. 3), number of grains per spike (Table 1 and Fig. 6), grain weight per spike (Table 1 and Fig. 7) and 1000 grain weight (Table 2 and Fig. 8). The decrease in number of grains per spike was main responsible factors for reducing the grain yield under stress condition. More reduction of grain yield per plant due to water stress and yield of the plants is intimately associated with the photosynthetic rate of the leaf area findings by Gifford and Evans, 1981; Baque, 2003). Similarly the decreased 1000 grains weight may be attributed to disturbed nutrient uptake efficiency and photosynthetic translocation within the plant that produced shrivelled grains due to hastened maturity. It is important to note that genotypes C-306, HI-1620, RW-5, MACS-6695, NIAW-3212, DBW-110, GW477 and NI-5439 which we believe have resistant to water deficit, had a feature of developmental plasticity and this enables them to produce seed on a limited supply of water which otherwise is coupled with the abundant of water (Quarrie et al., 1999). Similarly the other yield attributing parameters were also significantly influenced by differential water regimes and wheat genotypes.

The genotypes C-306, DBW-110, DBW-166, GW-477, HI-1620, MACS-6695, NIAW-3212 and RW-5 were attained early days to 50 per cent flowering and days to maturity (Table 1 and Fig. 4). The genotypes which flowered and matured earlier may have an advantage of allowing drought escape (Lopes et al., 2012) and have a capability to complete their life before dehydrated by high summer temperature, enabling the genotype to efficiently utilize rainfall during critical development stages (Fig. 5).

Maintenance of chlorophyll content is necessary for photosynthesis under moisture stress condition. Lower per cent of reduction and highest chlorophyll content under moisture stress in wheat tolerant genotype has been reported (Almeselmani, 2012; Kraus et al., 1995; Nyachiro et al., 2001). Similar result revealed that chlorophyll content decreased under drought stress (Meena Kumari et al., 2004).

Fig.1 Influence of WW and LMS on plant height in wheat genotypes

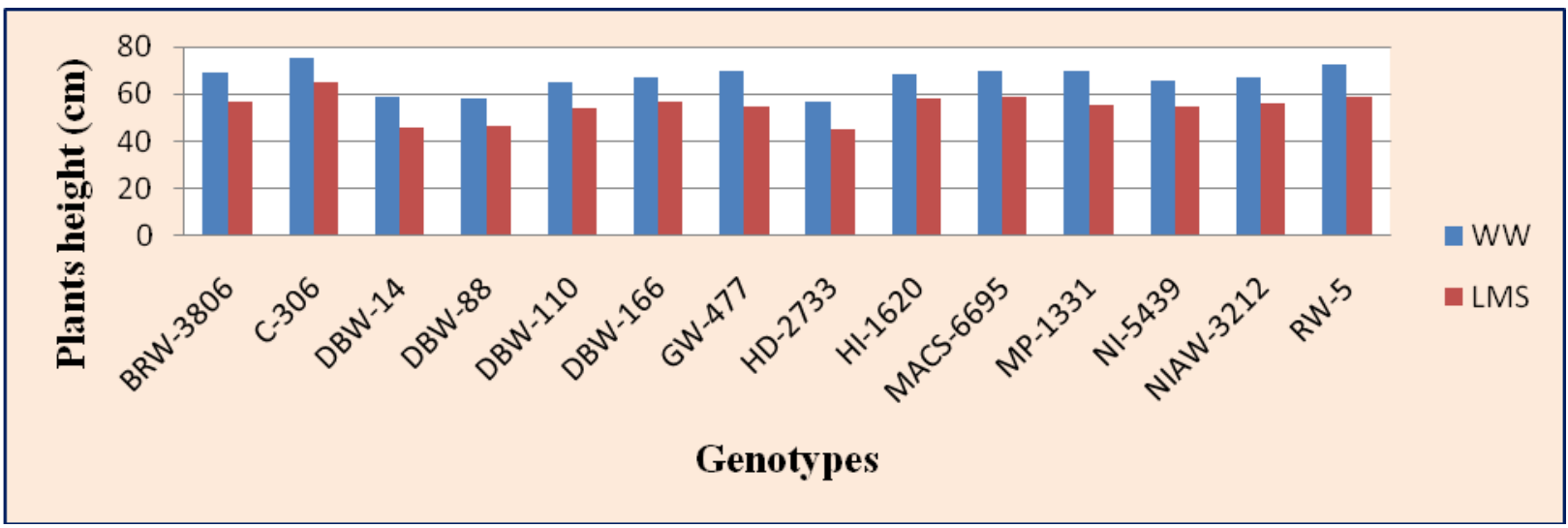


Fig.2 Influence of WW and LMS on No. of tillers per plant in wheat genotypes

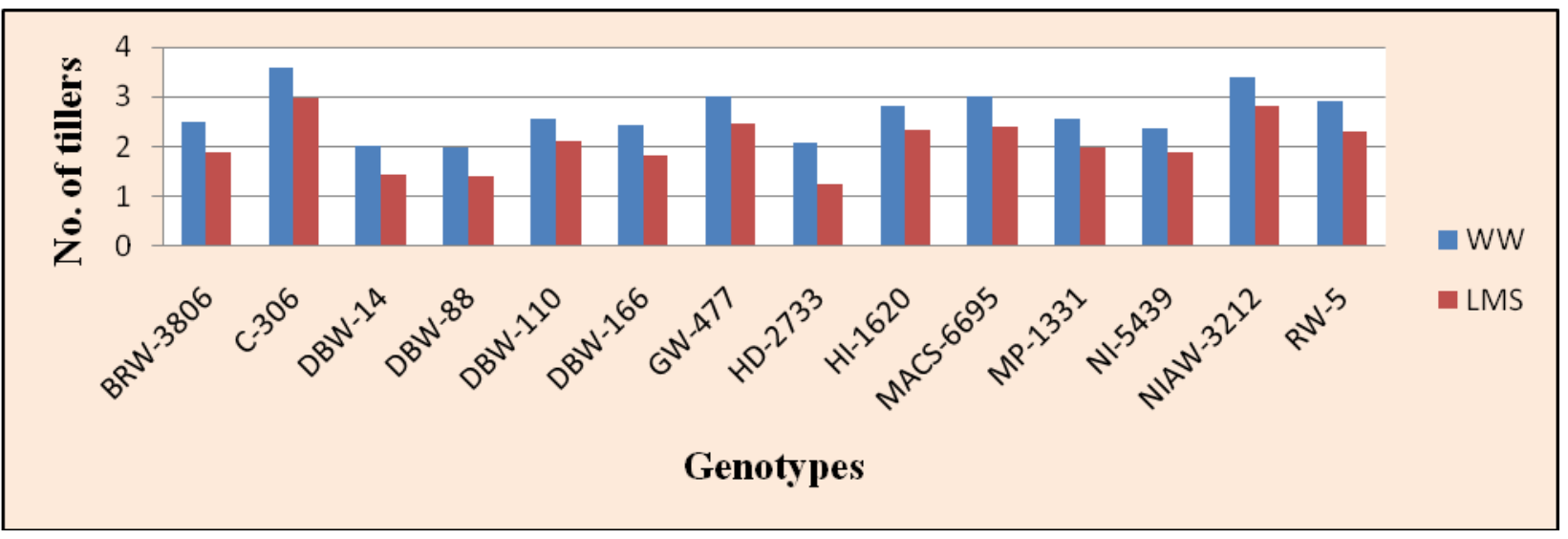

Fig.3 Influence of WW and LMS on spike length in wheat genotypes

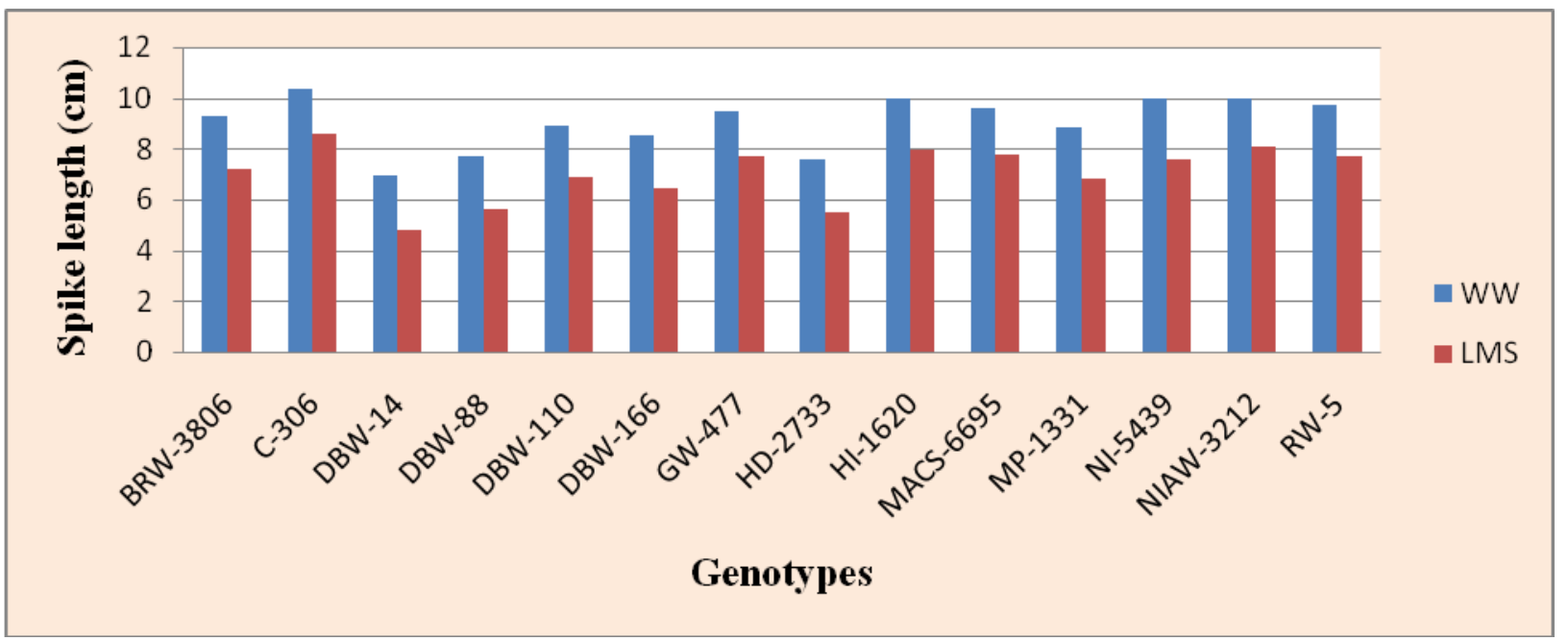

Fig.4 Influence of WW and LMS on days to $50 \%$ flowering in wheat genotypes

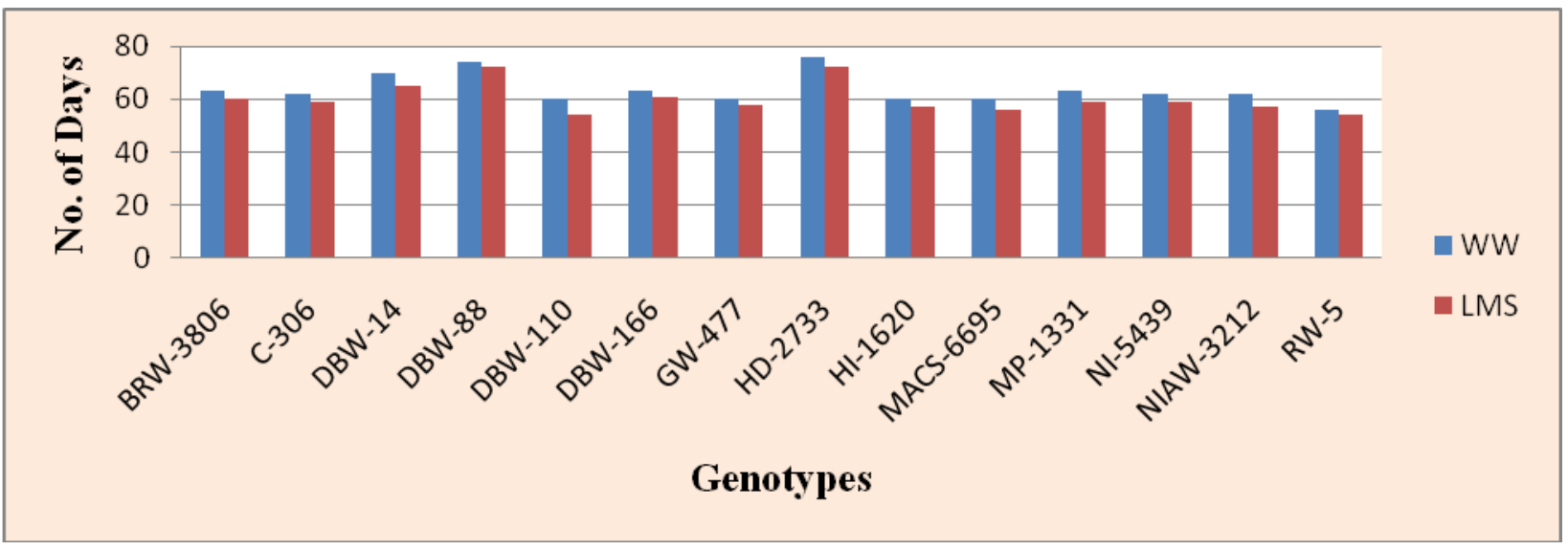


Fig.5 Influence of WW and LMS on days to maturity in wheat genotypes

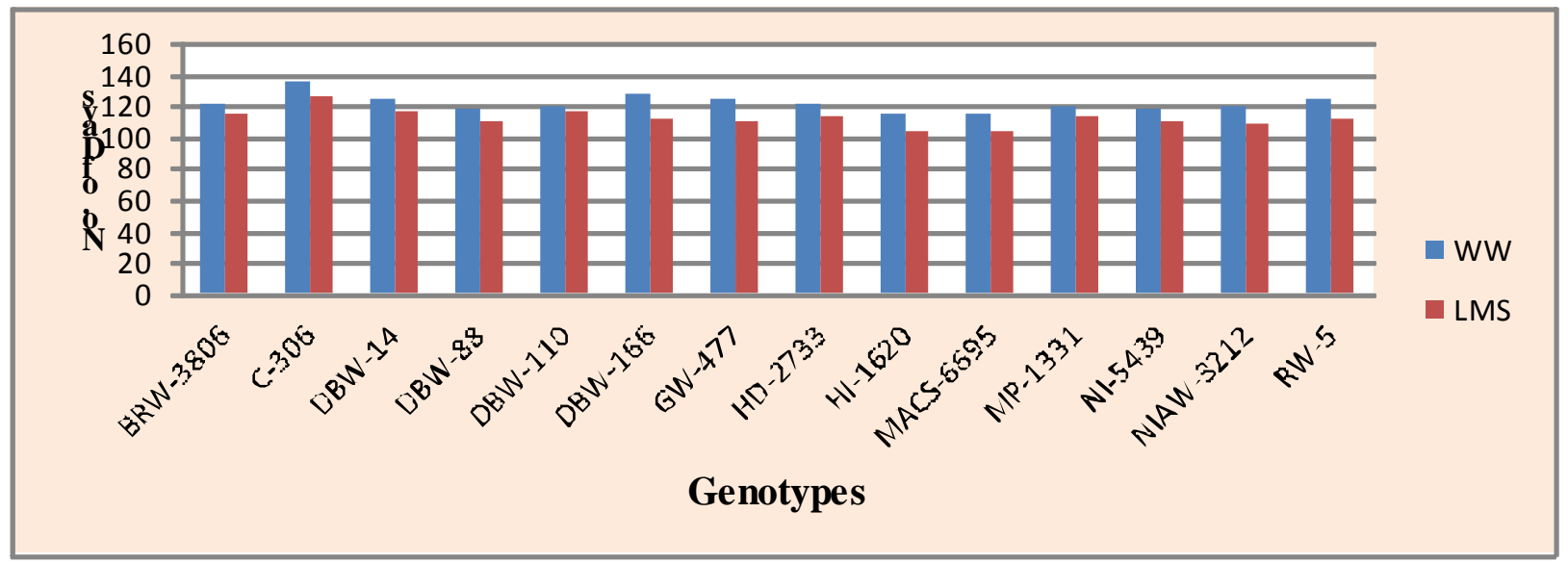

Fig.6 Influence of WW and LMS on No. of grains per spike in wheat genotypes

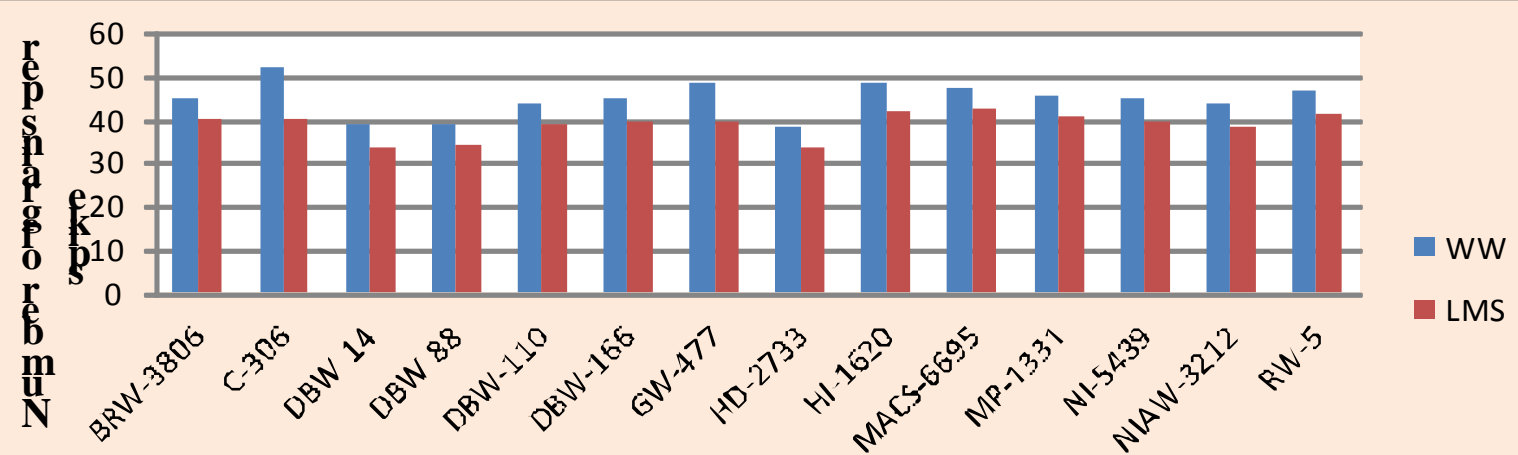

Genotypes

Fig.7 Influence of WW and LMS on grain weight per spike in wheat genotypes

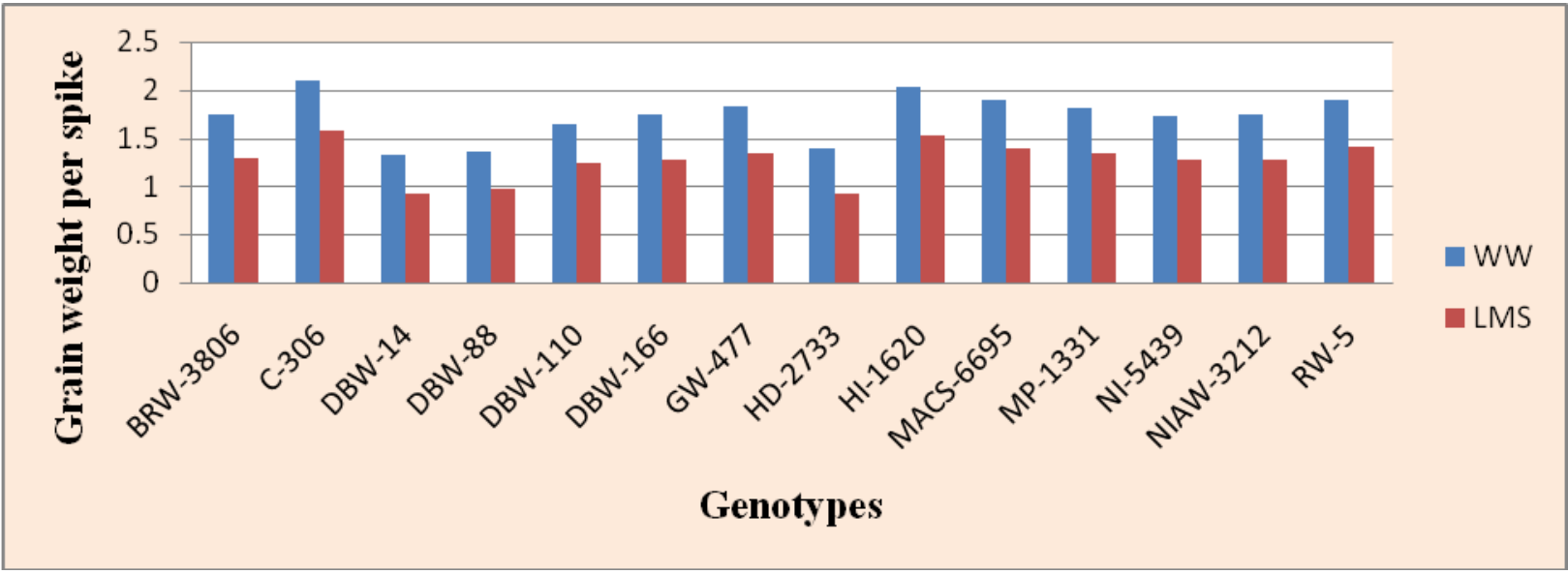


Fig.8 Influence of WW and LMS on 1000 grain in wheat genotypes

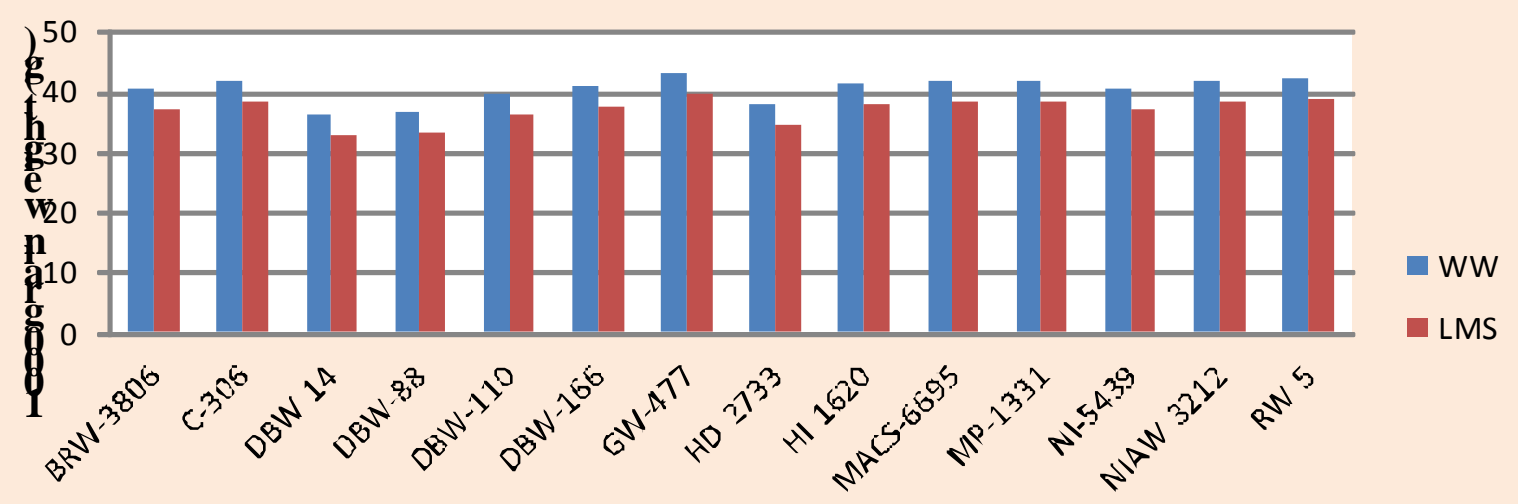

Genotypes

Fig.9 Influence of WW and LMS on dry matter accumulation in wheat genotypes

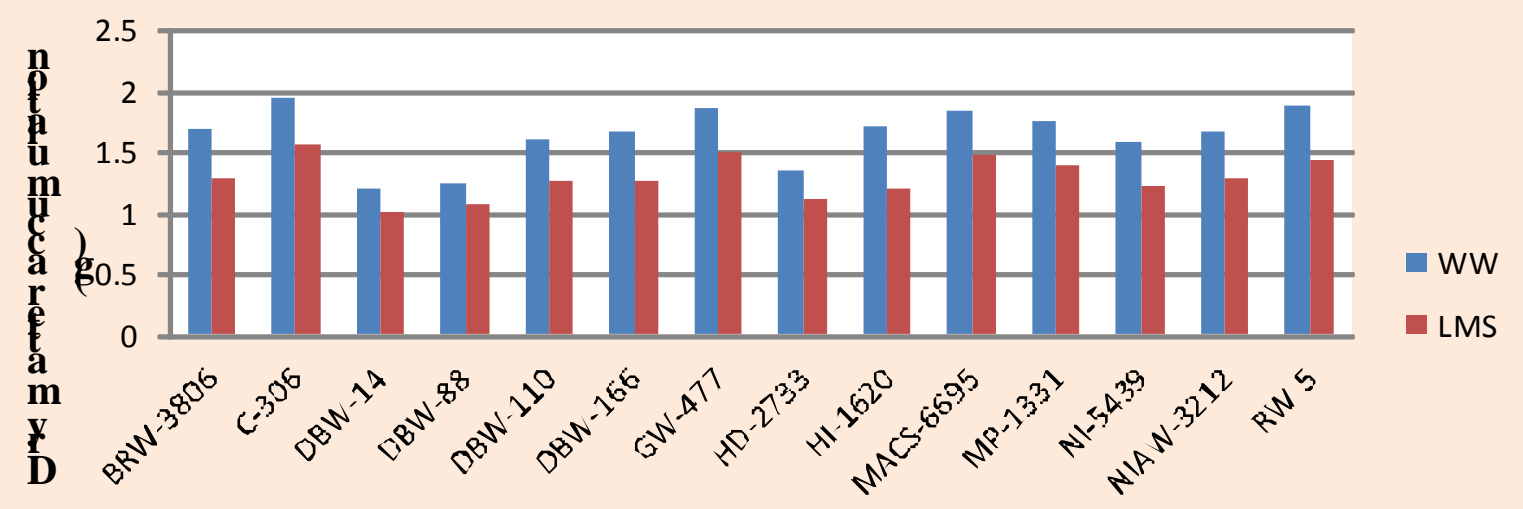

Genotypes 
Table.1 Yield quality parameters as influenced by WW and LMS

\begin{tabular}{|c|c|c|c|c|c|c|c|c|c|c|c|c|c|c|c|c|c|c|c|}
\hline \multirow[t]{2}{*}{$\begin{array}{l}\text { SI. } \\
\text { No. }\end{array}$} & \multirow[t]{2}{*}{ Genotypes } & \multicolumn{2}{|c|}{$\begin{array}{c}\text { Plant } \\
\text { height }(\mathrm{cm})\end{array}$} & \multicolumn{2}{|c|}{$\begin{array}{c}\text { Number of } \\
\text { tillers }\end{array}$} & \multicolumn{2}{|c|}{$\begin{array}{c}\text { Spike length } \\
\text { (cm) }\end{array}$} & \multicolumn{2}{|c|}{$\begin{array}{c}\text { Days to } 50 \\
\% \\
\text { flowering }\end{array}$} & \multicolumn{2}{|c|}{$\begin{array}{l}\text { Days to } \\
\text { maturity }\end{array}$} & \multicolumn{2}{|c|}{$\begin{array}{c}\text { SPAD } \\
\text { (30 DAS) }\end{array}$} & \multicolumn{2}{|c|}{$\begin{array}{c}\text { SPAD } \\
(60 \text { DAS })\end{array}$} & \multicolumn{2}{|c|}{$\begin{array}{c}\text { Number of } \\
\text { grains per } \\
\text { spike }\end{array}$} & \multicolumn{2}{|c|}{$\begin{array}{c}\text { Grain } \\
\text { weight per } \\
\text { spike (g) }\end{array}$} \\
\hline & & WW & LMS & WW & LMS & WW & LMS & WW & LMS & WW & LMS & WW & LMS & WW & LMS & WW & LMS & WW & LMS \\
\hline 1 & BRW-3806 & 68.9 & 56.7 & 2.50 & 1.90 & 9.27 & 7.20 & 63 & 60 & 122 & 115 & 44.63 & 41.17 & 55.93 & 54.37 & 45.17 & 40.00 & 1.75 & 1.29 \\
\hline 2 & C-306 & 75.2 & 64.8 & 3.57 & 2.97 & 10.37 & 8.57 & 62 & 59 & 135 & 126 & 47.80 & 45.21 & 56.47 & 55.30 & 51.89 & 40.10 & 2.09 & 1.58 \\
\hline 3 & DBW-14 & 58.9 & 45.9 & 2.03 & 1.43 & 6.93 & 4.83 & 70 & 65 & 125 & 116 & 41.97 & 39.54 & 43.54 & 40.58 & 38.67 & 33.83 & 1.32 & 0.92 \\
\hline 4 & DBW-88 & 58.1 & 46.3 & 2.00 & 1.40 & 7.70 & 5.60 & 74 & 72 & 118 & 110 & 38.50 & 36.90 & 56.30 & 54.00 & 39.00 & 34.07 & 1.36 & 0.97 \\
\hline 5 & DBW-110 & 65.2 & 54.3 & 2.56 & 2.10 & 8.90 & 6.87 & 60 & 54 & 120 & 117 & 44.00 & 41.23 & 54.03 & 53.60 & 43.60 & 38.83 & 1.65 & 1.24 \\
\hline 6 & DBW-166 & 67.1 & 56.9 & 2.43 & 1.83 & 8.53 & 6.45 & 63 & 61 & 128 & 112 & 46.70 & 44.80 & 43.57 & 42.50 & 44.70 & 39.50 & 1.74 & 1.28 \\
\hline 7 & GW-477 & 69.9 & 54.4 & 3.00 & 2.47 & 9.50 & 7.73 & 60 & 58 & 124 & 110 & 45.77 & 44.43 & 54.33 & 52.97 & 48.33 & 39.37 & 1.83 & 1.35 \\
\hline 8 & HD-2733 & 57.0 & 45.4 & 2.07 & 1.25 & 7.57 & 5.50 & 76 & 72 & 122 & 113 & 35.20 & 35.18 & 45.03 & 43.30 & 38.33 & 33.43 & 1.39 & 0.92 \\
\hline 9 & HI-1620 & 68.5 & 57.8 & 2.83 & 2.33 & 10.00 & 7.93 & 60 & 57 & 115 & 104 & 45.90 & 44.53 & 54.40 & 53.93 & 48.67 & 42.20 & 2.03 & 1.52 \\
\hline 10 & HI- 1628 & 63.6 & 53.4 & 2.50 & 1.90 & 8.60 & 6.57 & 64 & 59 & 122 & 113 & 42.20 & 42.97 & 50.77 & 49.77 & 43.00 & 36.07 & 1.58 & 1.13 \\
\hline 11 & HW-1235 & 60.1 & 49.8 & 2.40 & 1.80 & 9.00 & 6.57 & 62 & 55 & 117 & 112 & 41.70 & 41.77 & 47.77 & 49.17 & 41.33 & 36.20 & 1.49 & 1.07 \\
\hline 12 & JWS-810 & 63.1 & 52.4 & 2.80 & 1.90 & 8.40 & 6.33 & 64 & 60 & 115 & 106 & 42.90 & 41.87 & 48.67 & 49.90 & 43.00 & 38.10 & 1.56 & 1.12 \\
\hline 13 & K-1317 & 62.0 & 52.8 & 2.60 & 1.70 & 8.20 & 6.17 & 71 & 67 & 124 & 115 & 40.07 & 40.10 & 50.57 & 49.97 & 43.33 & 38.03 & 1.59 & 1.11 \\
\hline 14 & M-516 & 60.8 & 51.3 & 2.73 & 2.13 & 8.49 & 6.62 & 65 & 59 & 115 & 112 & 38.80 & 39.23 & 50.07 & 49.03 & 43.67 & 38.70 & 1.54 & 1.11 \\
\hline 15 & MACS-6695 & 70.0 & 59.0 & 3.00 & 2.40 & 9.60 & 7.77 & 60 & 56 & 115 & 104 & 47.30 & 44.63 & 55.40 & 52.33 & 47.38 & 42.41 & 1.90 & 1.41 \\
\hline 16 & MACS-6696 & 64.2 & 52.4 & 2.13 & 1.60 & 8.58 & 6.61 & 67 & 64 & 117 & 108 & 45.03 & 42.43 & 49.87 & 49.23 & 42.67 & 37.73 & 1.59 & 1.13 \\
\hline 17 & MP-1331 & 69.5 & 55.7 & 2.57 & 1.97 & 8.87 & 6.83 & 63 & 59 & 120 & 114 & 47.03 & 44.57 & 54.32 & 53.10 & 45.33 & 40.50 & 1.82 & 1.34 \\
\hline
\end{tabular}


Int.J.Curr.Microbiol.App.Sci (2019) 8(11): 2395-2407

\begin{tabular}{|c|c|c|c|c|c|c|c|c|c|c|c|c|c|c|c|c|c|c|c|}
\hline 18 & MP-3288 & 64.2 & 51.6 & 2.00 & 1.54 & 8.20 & 6.60 & 66 & 62 & 121 & 112 & 41.43 & 37.13 & 50.43 & 47.57 & 41.67 & 36.83 & 1.53 & 1.10 \\
\hline 19 & NI-5439 & 65.3 & 54.8 & 2.37 & 1.90 & 10.01 & 7.61 & 62 & 59 & 119 & 110 & 44.97 & 41.37 & 56.03 & 54.90 & 44.81 & 39.78 & 1.73 & 1.28 \\
\hline 20 & NIAW-3170 & 64.2 & 51.1 & 2.63 & 2.03 & 7.82 & 5.79 & 65 & 60 & 122 & 113 & 42.70 & 40.27 & 50.00 & 48.67 & 42.67 & 33.67 & 1.45 & 1.02 \\
\hline 21 & NIAW-3212 & 67.2 & 55.9 & 3.40 & 2.80 & 10.01 & 8.08 & 62 & 57 & 120 & 108 & 44.67 & 41.13 & 54.10 & 53.17 & 43.67 & 38.50 & 1.74 & 1.28 \\
\hline 22 & RIL-SI-38 & 64.3 & 54.9 & 2.20 & 1.33 & 8.47 & 6.09 & 64 & 61 & 122 & 113 & 45.50 & 40.03 & 54.00 & 48.41 & 44.28 & 37.51 & 1.66 & 1.18 \\
\hline 23 & RW-5 & 72.4 & 59.0 & 2.90 & 2.30 & 9.72 & 7.70 & 56 & 54 & 124 & 112 & 46.80 & 41.98 & 53.60 & 52.33 & 46.89 & 41.29 & 1.89 & 1.41 \\
\hline 24 & UAS-347 & 60.2 & 50.0 & 2.23 & 1.63 & 8.23 & 6.36 & 65 & 60 & 125 & 109 & 43.44 & 39.33 & 47.97 & 45.30 & 42.67 & 38.03 & 1.49 & 1.07 \\
\hline 25 & UAS-375 & 60.1 & 49.2 & 2.07 & 1.47 & 8.37 & 6.33 & 64 & 60 & 110 & 103 & 43.17 & 39.53 & 49.00 & 43.97 & 39.44 & 37.27 & 1.40 & 1.09 \\
\hline 26 & UAS-446 & 63.7 & 50.6 & 2.20 & 1.47 & 8.30 & 6.47 & 63 & 61 & 110 & 100 & 42.03 & 38.20 & 50.90 & 44.23 & 40.11 & 37.23 & 1.38 & 1.08 \\
\hline & Mean & 64.8 & 53.3 & 2.53 & 1.91 & 8.75 & 6.74 & 64 & 60 & 120 & 111 & 43.47 & 41.14 & 51.43 & 49.83 & 43.63 & 38.05 & 1.64 & 1.19 \\
\hline & S.Em \pm & 3.31 & 2.98 & 0.21 & 0.10 & 0.29 & 0.28 & 1.81 & 1.66 & 3.07 & 2.97 & 2.01 & 1.39 & 1.77 & 1.71 & 1.50 & 1.12 & 0.08 & 0.07 \\
\hline \multicolumn{2}{|c|}{ C.D. $(P=0.05)$} & 9.39 & 8.47 & 0.59 & 0.40 & 0.81 & 0.80 & 5.13 & 4.72 & 8.73 & 8.44 & 5.71 & 3.94 & 5.03 & 4.85 & 4.25 & 3.18 & 0.23 & 0.20 \\
\hline
\end{tabular}

Note: WW- Well water, LMS- Low Moisture Stress 
Table.2 Yield quality parameters as influenced by WW and LMS

\begin{tabular}{|c|c|c|c|c|c|c|c|}
\hline \multirow[t]{2}{*}{ SI. No. } & \multirow[t]{2}{*}{ Genotypes } & \multicolumn{2}{|c|}{1000 grain weight $(\mathrm{g})$} & \multicolumn{2}{|c|}{ Dry matter accumulation per plant (g) } & \multicolumn{2}{|c|}{ Grain yield } \\
\hline & & WW & LMS & WW & LMS & WW & LMS \\
\hline 1 & BRW-3806 & 40.59 & 37.09 & 1.69 & 1.29 & 4.56 & 1.78 \\
\hline 2 & C-306 & 41.61 & 38.11 & 1.94 & 1.55 & 5.45 & 2.02 \\
\hline 3 & DBW-14 & 36.03 & 32.53 & 1.20 & 1.01 & 3.44 & 1.39 \\
\hline 4 & DBW-88 & 36.64 & 33.14 & 1.24 & 1.07 & 3.54 & 1.57 \\
\hline 5 & DBW-110 & 39.58 & 36.08 & 1.61 & 1.25 & 4.29 & 1.97 \\
\hline 6 & DBW-166 & 40.78 & 37.28 & 1.67 & 1.26 & 4.53 & 1.91 \\
\hline 7 & GW-477 & 43.02 & 39.52 & 1.86 & 1.50 & 4.76 & 1.93 \\
\hline 8 & HD-2733 & 37.91 & 34.41 & 1.35 & 1.11 & 3.60 & 1.34 \\
\hline 9 & HI-1620 & 41.50 & 38.00 & 1.71 & 1.20 & 5.27 & 2.03 \\
\hline 10 & HI- 1628 & 38.48 & 34.98 & 1.29 & 1.13 & 4.11 & 1.73 \\
\hline 11 & HW-1235 & 37.73 & 34.23 & 1.34 & 1.23 & 3.86 & 1.67 \\
\hline 12 & JWS-810 & 38.04 & 34.54 & 1.39 & 1.03 & 4.06 & 1.72 \\
\hline 13 & K-1317 & 38.61 & 35.11 & 1.42 & 1.06 & 4.14 & 1.64 \\
\hline 14 & M-516 & 37.12 & 33.62 & 1.46 & 1.23 & 4.01 & 1.71 \\
\hline 15 & MACS-6695 & 41.96 & 38.46 & 1.83 & 1.47 & 4.95 & 2.00 \\
\hline 16 & MACS-6696 & 39.08 & 35.58 & 1.29 & 1.07 & 4.13 & 1.73 \\
\hline 17 & MP-1331 & 41.84 & 38.34 & 1.74 & 1.38 & 4.72 & 1.86 \\
\hline 18 & MP-3288 & 38.51 & 35.01 & 1.43 & 1.11 & 3.98 & 1.70 \\
\hline 19 & NI-5439 & 40.44 & 36.94 & 1.57 & 1.21 & 4.50 & 1.94 \\
\hline 20 & NIAW-3170 & 37.83 & 34.33 & 1.41 & 1.05 & 3.78 & 1.62 \\
\hline 21 & NIAW-3212 & 41.70 & 38.20 & 1.66 & 1.28 & 4.53 & 1.88 \\
\hline 22 & RIL-SI-38 & 39.14 & 35.64 & 1.40 & 1.04 & 4.30 & 1.71 \\
\hline 23 & RW-5 & 42.09 & 38.59 & 1.88 & 1.43 & 4.92 & 1.92 \\
\hline 24 & UAS-347 & 37.81 & 34.31 & 1.34 & 1.04 & 3.88 & 1.67 \\
\hline 25 & UAS-375 & 37.41 & 33.91 & 1.44 & 1.08 & 3.65 & 1.69 \\
\hline 26 & UAS-446 & 38.21 & 34.71 & 1.41 & 1.11 & 3.60 & 1.63 \\
\hline \multicolumn{2}{|c|}{ Mean } & 39.37 & 35.87 & 1.52 & 1.20 & 4.25 & 1.76 \\
\hline \multicolumn{2}{|c|}{ S.Em \pm} & 1.30 & 2.13 & 0.09 & 0.08 & 0.21 & 0.08 \\
\hline \multicolumn{2}{|c|}{ C.D. $(P=0.05)$} & 3.69 & 3.55 & 0.25 & 0.24 & 0.59 & 0.23 \\
\hline
\end{tabular}

Note: WW- Well water, LMS- Low Moisture Stress 
Table.3 Seed quality parameters as influenced by WW and LMS

\begin{tabular}{|c|c|c|c|c|c|c|c|c|c|c|c|c|c|c|c|c|c|}
\hline \multirow[t]{2}{*}{$\begin{array}{l}\text { SI. } \\
\text { No. }\end{array}$} & \multirow[t]{2}{*}{ Genotypes } & \multicolumn{2}{|c|}{ Germination percentage } & \multicolumn{2}{|c|}{ Root length } & \multicolumn{2}{|c|}{ Shoot length } & \multicolumn{2}{|c|}{$\begin{array}{l}\text { Seedling } \\
\text { length }(\mathrm{cm})\end{array}$} & \multicolumn{2}{|c|}{$\begin{array}{l}\text { Seedling dry } \\
\text { weight (mg0 }\end{array}$} & \multicolumn{2}{|c|}{$\begin{array}{l}\text { Seedling } \\
\text { vigour index } \\
\qquad \text { I }\end{array}$} & \multicolumn{2}{|c|}{$\begin{array}{l}\text { Seedling vigour } \\
\text { index II }\end{array}$} & \multicolumn{2}{|c|}{$\mathrm{EC}\left(\mathrm{dSm}^{-1}\right)$} \\
\hline & & WW & LMS & WW & LMS & WW & LMS & WW & LMS & WW & LMS & WW & LMS & WW & LMS & WW & LMS \\
\hline 1 & BRW-3806 & $95.7(78.0) *$ & $93.3(75.0)$ & 6.9 & 5.4 & 7.4 & 5.8 & 14.4 & 11.4 & 307.33 & 293.53 & 1375 & 1060 & 29 & 27 & 0.341 & 0.483 \\
\hline 2 & C-306 & $96.8(79.7)$ & $91.5(73.0)$ & 7.0 & 5.2 & 7.3 & 5.9 & 14.2 & 11.1 & 309.33 & 295.53 & 1375 & 1024 & 30 & 27 & 0.363 & 0.473 \\
\hline 3 & DBW-14 & $86.6(68.5)$ & $81.5(64.5)$ & 5.3 & 3.8 & 4.3 & 4.0 & 9.7 & 7.9 & 228.00 & 214.20 & 836 & 642 & 20 & 17 & 0.434 & 0.544 \\
\hline 4 & DBW-88 & $86.2(68.2)$ & $85.0(75.3)$ & 4.8 & 3.3 & 4.7 & 3.5 & 9.5 & 6.8 & 270.33 & 256.53 & 817 & 577 & 23 & 22 & 0.426 & 0.536 \\
\hline 5 & DBW-110 & $96.2(78.7)$ & $93.6(72.0)$ & 7.2 & 5.9 & 7.1 & 5.6 & 14.2 & 11.5 & 305.67 & 291.87 & 1368 & 1050 & 29 & 27 & 0.358 & 0.468 \\
\hline 6 & DBW-166 & $95.7(78.0)$ & $90.4(67.2)$ & 8.4 & 6.7 & 7.2 & 5.7 & 15.5 & 12.5 & 308.00 & 294.20 & 1480 & 1127 & 29 & 27 & 0.322 & 0.471 \\
\hline 7 & GW-477 & $95.9(78.3)$ & $93.9(75.7)$ & 6.2 & 4.8 & 7.8 & 6.3 & 14.1 & 11.5 & 312.00 & 298.20 & 1349 & 1039 & 30 & 28 & 0.337 & 0.462 \\
\hline 8 & HD-2733 & $83.0(65.6)$ & 80.7 (63.9) & 5.1 & 3.7 & 4.4 & 3.0 & 9.4 & 6.7 & 262.33 & 248.53 & 779 & 541 & 22 & 20 & 0.433 & 0.543 \\
\hline 9 & HI-1620 & 96.9 (79.9) & $95.8(78.2)$ & 7.5 & 6.0 & 7.0 & 5.5 & 14.4 & 11.2 & 306.83 & 279.07 & 1399 & 1095 & 30 & 27 & 0.347 & 0.457 \\
\hline 10 & HI-1628 & $85.6(67.7)$ & $83.8(66.2)$ & 6.6 & 5.1 & 6.0 & 4.5 & 12.6 & 9.6 & 256.00 & 242.20 & 1080 & 806 & 22 & 20 & 0.404 & 0.514 \\
\hline 11 & HW-1235 & $91.5(73.1)$ & $85.4(67.5)$ & 6.4 & 4.9 & 6.1 & 4.4 & 12.6 & 9.4 & 291.67 & 283.53 & 1150 & 800 & 27 & 24 & 0.419 & 0.529 \\
\hline 12 & JWS-810 & $94.5(76.4)$ & $85.8(67.8)$ & 6.5 & 5.0 & 6.3 & 4.8 & 12.9 & 9.9 & & 230.87 & 1215 & 846 & 29 & 20 & 0.403 & 0.513 \\
\hline 13 & K-1317 & $88.1(69.8)$ & $86.3(68.3)$ & 6.6 & 5.1 & 6.1 & 4.3 & 12.7 & 9.4 & 291.00 & 277.20 & 1121 & 809 & 26 & 24 & 0.386 & 0.496 \\
\hline 14 & M-516 & $91.0(72.6)$ & $86.7(68.6)$ & 6.3 & 4.8 & 6.0 & 4.4 & 12.3 & 9.3 & 243.67 & 229.87 & 1121 & 805 & 22 & 20 & 0.380 & 0.490 \\
\hline 15 & MACS-6695 & $95.2(77.3)$ & 89.7 (71.2) & 7.0 & 5.5 & 7.5 & 6.3 & 14.5 & 11.7 & 317.00 & 305.87 & 1380 & 1051 & 31 & 27 & 0.360 & 0.470 \\
\hline 16 & MACS-6696 & 91.7 (73.3) & $84.2(66.6)$ & 6.5 & 5.0 & 6.0 & 4.2 & 12.5 & 9.2 & 297.33 & 276.20 & 1150 & 777 & 27 & 23 & 0.389 & 0.499 \\
\hline 17 & MP-1331 & 95.3 (77.5) & $91.1(72.6)$ & 6.6 & 5.1 & 6.8 & 5.5 & 13.4 & 10.6 & 293.60 & 292.53 & 1279 & 959 & 28 & 27 & 0.369 & 0.479 \\
\hline 18 & MP-3288 & $87.2(69.0)$ & $84.5(66.8)$ & 6.5 & 5.0 & 5.6 & 3.9 & 12.2 & 9.0 & 306.33 & 225.53 & 1060 & 756 & 27 & 19 & 0.389 & 0.499 \\
\hline 19 & NI-5439 & $95.6(77.8)$ & $93.0(74.7)$ & 6.7 & 5.2 & 7.6 & 6.1 & 14.3 & 11.3 & 257.67 & 243.87 & 1371 & 1055 & 25 & 23 & 0.337 & 0.447 \\
\hline 20 & NIAW-3170 & $86.4(68.4)$ & $85.3(67.5)$ & 6.0 & 4.5 & 5.5 & 4.5 & 11.5 & 9.0 & 291.00 & 239.53 & 989 & 756 & 25 & 21 & 0.408 & 0.518 \\
\hline 21 & NIAW-3212 & $96.4(79.1)$ & $93.6(75.3)$ & 7.4 & 5.9 & 7.8 & 5.8 & 15.2 & 11.7 & 311.20 & 294.13 & 1462 & 1096 & 30 & 28 & 0.364 & 0.474 \\
\hline 22 & RIL-SI-38 & $92.5(74.1)$ & 89.5 (71.1) & 6.1 & 4.6 & 6.2 & 4.7 & 12.3 & 9.3 & 294.33 & 236.20 & 1136 & 831 & 27 & 21 & 0.413 & 0.523 \\
\hline 23 & RW-5 & $95.9(78.4)$ & $90.6(72.1)$ & 7.8 & 6.3 & 7.0 & 5.5 & 14.8 & 11.8 & 310.00 & 276.73 & 1421 & 1070 & 30 & 25 & 0.365 & 0.475 \\
\hline 24 & UAS-347 & $91.8(73.3)$ & $85.0(67.2)$ & 6.5 & 5.0 & 5.7 & 5.1 & 12.1 & 10.1 & 240.33 & 215.87 & 1114 & 857 & 22 & 18 & 0.387 & 0.497 \\
\hline 25 & UAS-375 & $91.2(72.7)$ & $84.3(66.7)$ & 6.2 & 5.0 & 5.9 & 4.7 & 12.1 & 9.7 & 255.67 & 241.87 & 1104 & 821 & 23 & 20 & 0.360 & 0.470 \\
\hline 26 & UAS-446 & $90.5(72.0)$ & $85.2(67.4)$ & 6.3 & 5.0 & 6.0 & 4.6 & 12.3 & 9.6 & 234.00 & 220.47 & 1116 & 819 & 21 & 19 & 0.385 & 0.495 \\
\hline \multicolumn{2}{|c|}{ Mean } & $92.1(73.6)$ & $88.1(69.8)$ & 6.5 & 5.1 & 6.4 & 5.0 & 12.9 & 10.0 & 284.91 & 261.70 & 1194 & 887 & 26 & 23 & 0.380 & 0.493 \\
\hline \multicolumn{2}{|c|}{ S.Em \pm} & 1.45 & 1.18 & 0.43 & 0.42 & 0.50 & 0.29 & 0.67 & 0.53 & 17.62 & 16.84 & 58.72 & 43.51 & 1.63 & 1.60 & 0.02 & 0.02 \\
\hline \multicolumn{2}{|c|}{ C.D. $(P=0.05)$} & 6.10 & 5.0 & 1.76 & 1.78 & 2.12 & 1.23 & 2.83 & 2.23 & 74.19 & 70.91 & 247.1 & 183.1 & 6.86 & 6.75 & 0.09 & 0.09 \\
\hline
\end{tabular}




\section{Seed quality parameters}

Seed quality parameters after the harvest of the wheat crop were also significantly influenced by two moisture regimes. The wheat seeds of 26 genotypes from two moisture condition were significantly high in seedling vigour index I. The seeds from well water situation greater seedling vigour index I was noticed in the genotype DBW-166 (1480) followed by NIAW-3212 (1462) while, HD2733 (779) noticed lowest. The seeds from stress condition, DBW-110 (1127) recorded highest SVI followed by NIAW-3212 (1096) while compared to DBW-88 (541). The seedling vigour index II was found significant in all the genotypes between two moisture regimes. An average seedling vigour index II was 26 and 23 in well water and stress condition respectively. As the seeds from well water situation greatest seedling vigour index II was recorded in the genotype MACS-6695 (31) followed by NIAW-3212 (30). While, the genotype DBW-14 (20) noticed lowest value of seedling vigour index II. As the seeds from stress condition, the genotype GW-477 (28) and NIAW-3212 (28) recorded highest seedling vigour index II followed by while compared to DBW-14 (17) (Table 3).

The increased in seedling vigour index I and II may be attributed higher germination percentage, root length, shoot length, seedling length and seedling dry weight in well water as well as in low moisture condition.

The seedling vigour index was reduced in low moisture condition as compared to well water condition. This may be due to eventual depletion of grain moisture which produces smaller endosperm and premature seed with potentially reduced germination percentage and seedling vigour. Similar findings were stated by Saini and Westagate (2000). The decrease in the shoot length, root length and seedling length might be due to a difficulty of elongation and cell division leading to type of tuberization. This lignification and the tuberization of the root system allow the plant to enter a slow-down state, while waiting for the conditions to become favourable again (Fraser et al., 1990). Moisture stress probably perturbed the physiological mechanisms of the sensitive varieties more severely than the tolerant varieties. The seeds under stress decreased as compared to well water due to shriveled seeds and nutritive value lower and may be due to a difficulty of elongation and cell division. Similar findings supported by Saini and Westagate (2000). The decline in seedling dry weight was attributed due to length of the seedling was lesser with increased osmotic stress level finding was reported by Ming et al., (2012); Moucheshi et al., (2012); Almadi et al., (2012).

The electrical conductivity was significant in all the genotypes between two moisture regimes .All the genotypes of wheat from different moisture regimes found highly significant on electrical conductivity. The seeds from well water condition lowest electrical conductivity was recorded in the genotype DBW-166 $\left(0.322 \mathrm{dSm}^{-1}\right)$ while the highest value of electrical conductivity was recorded for the genotype DBW-14 (0.434 $\left.\mathrm{dSm}^{-1}\right)$. As the seeds from stress condition the lowest value of electrical conductivity was found in the genotype GW-477 $\left(0.462 \mathrm{dSm}^{-1}\right)$ and premier value of electrical conductivity was noticed in DBW-14 $\left(0.544 \mathrm{dSm}^{-1}\right)$. The genotypes NI-5439, HI-1620, GW-477, C306, DBW-110 and NIAW-3212 had maximum membrane stability index. Higher membrane stability reflects the existence of stress resistance mechanism in these genotypes. The results from electrolyte leakage measurements showed that membrane integrity was conserved for tolerant as compared to susceptible genotypes which are in supported Almeselmani et al., (2012) that electrolyte leakage was related with drought tolerance. 
In conclusion, the growth and yield attributing parameters such as days to 50 per cent flowering, days to maturity, plant height $(\mathrm{cm})$, number of productive tillers per plant, number of grains per pike, 1000 grain weight $(\mathrm{g})$, spike length $(\mathrm{cm})$, grain weight per spike, SPAD chlorophyll meter reading and grain yield showed that the genotypes were highly significant for all the traits under well water condition as compared to drought indicating that the genotypes performed differentially from each other for all the traits. Finally, the genotypes C-306, BRW-3806, GW-477, HI1620, MACS-6695, MP-1331, NIAW-3212, NI-5439 and RW-5 were found to have better combination of above mentioned characters and maximum yield was observed when compared to all the genotypes.

\section{References}

Almadi, G., Akbarabadi, A., Kahrizi, D., Rezaizad, A. and Gheytouli, M. 2012, Study of drought tolerance of bread wheat (Triticum aestivum L.) genotypes in seedling stage. Biharean Biol., 6(2): 77-80.

Almeselmani, M., Saud, A. A., Al-Zubi, K., Hareri, F., Ammar, A. M., Kanbar, O. Z., Naseef, H., Al-nator, A. and Alsaed, H. (2012) Physiological attributes associated to water deficit tolerance of Syrian durum wheat varieties. Experimental Agriculture and Horticulture 1, 21-41.

Baque, M. A. (2003) Potassium induced changes in the physiology of wheat plants (Triticum aestivum L.) under water stress conditions. M.Sc.(Agri) Thesis, Bangabandhu Sheikh Mujibur Rahman Agril. Univ. Gazipur.

Fraser, T., Silk, W. and Rosr, T. (1990) Effect of low water potential on cortical cell length in growing region on maize roots. Plant Physiol., 93: 648-651.

Gifford, R. M. and Evans, L. T. (1981)
Photosynthesis, carbon partitioning and yield. Annu. Rev. Plant Physiology 32, 485-509.

Joshi, A. K., Mishra, B., Chatttarth, R., Ortiz, F. G. and Singh, R. P. (2007) Wheat improvement in India, preset status, emerging challenges and future prospects. Euphytica, 157(1), 431-446.

Kraus, C., Moutonnet, P., Hera, C. and Nielsen, D. R. (1995) Crop yield response to deficit irrigation. Kluwer Academic publishers, New York, 3344.

Lopes, M. S., Reynolds, M. P., Jalal-kamali, M. R., Moussa, K. S., Feltaous, MY., Tahir, I. S. A., Barma, N., Vargas, M. Y., Mannes, M. Y. and Baum, M., 2012, The yield correlations of selectable physiological traits in a population of advanced spring wheat lines grown in warm and drought environments. Field Crops Res., 128: 19-136.

Meena Kumari, Sain Dass, Vimala, Y. and Arora, P. (2004) Physiological parameters governing drought tolerance in maize. Indian Journal of Plant Physiology 9(2), 203-207.

Ming, D.F., Pei, M. S., Naeem, H. J., Gong, K. and Zhan, W. J. (2012) Silicon alleviates PEG- induced water-deficit stress in upland rice seedling by enhancing osmotic adjustment. $J$. Agron. Crop Sci., 198:14-26.

Moucheshi, A. B., Heidari, B. and Assad, M. T. (2012) Alleviation of drought stress effects on wheat using arbuscular mycorrhizal symbiosis. Int. J. Agric Sci., 291:35-47.

Nyachiro, J. M., Briggs, K. G., Hoddinott, J. and Johnson-Flanagan, A. M. (2001) Chlorophyll content, chlorophyll fluorescence and water deficit in spring wheat. Cereal Research. Communication 29, 135-142.

Quarrie, S. A., Stojanovic, J. and Pekic, S. 
(1999) Improving drought resistance in small-grained cereals: A case study, progress and prospects. Plant growth Regulator 29, 1-21.
Reproductive development in grain crops during drought. Elsevier: San Diego, 68: 59-96.

Saini, H. S. and Westagate, M. E. (2000)

\section{How to cite this article:}

Vidyashree S. Naduvinamani, S.B. Patil, Ashok S. Sajjan and Patil, M.D. 2019. Screening of wheat (Triticum aestivum L.) genotypes for field performance and seed quality parameters. Int.J.Curr.Microbiol.App.Sci. 8(11): 2395-2407. doi: https://doi.org/10.20546/ijcmas.2019.811.278 